Magdalena Słowik

Uniwersytet Jagielloński

magdalena.slowik1000@gmail.com

\title{
Aspectos semántico-pragmáticos de los diminutivos en el español de la Ciudad de México
}

\begin{abstract}
Resumen:
El tema del presente trabajo se centra en el análisis del fenómeno de la sufijación diminutiva en el español de la Ciudad de México. La parte práctica viene precedida de diversas consideraciones teóricas. El objetivo principal de las investigaciones, llevadas a cabo a partir del corpus de Internet Corpus Sociolingüístico de la Ciudad de México, es la identificación de las funciones de los diminutivos empleados en diferentes contextos situacionales. El proceso de investigación parte de enunciados de mexicanos de entre 20 y 34 años. Asimismo, en el análisis realizado, además de los factores lingüísticos, se han tenido en cuenta también factores sociolingüísticos como el sexo y el nivel de instrucción de los informantes.
\end{abstract}

Palabras clave: español de México, diminutivos, valores de los diminutivos, pragmática, sociolingüística

\begin{abstract}
:
Pragmatic and Sociolinguistic Aspects of Diminutives in the Variety of Spanish Spoken in Mexico City

The subject matter of the article is the analysis of suffixation of diminutives in the variety of Spanish spoken in Mexico City. The practical part is preceded by theoretical considerations. The aim of the study, based on an electronic corpus (Corpus Sociolingüístico de la Ciudad de México), is the identification of the functions of diminutives used in different situational contexts. The study focuses on statements made by Mexicans at the age of 20-34. Apart from linguistic
\end{abstract}


factors, the sociolinguistic aspects such as sex and education level of the informants are analysed.

Keywords: Mexican Spanish, diminutives, functions, pragmatics, sociolinguistics

\section{Introducción}

Los medios gramaticales existentes en el sistema lingüístico permiten al hablante expresar emociones y sentimientos, tanto positivos como negativos. Uno de los medios para expresar la afectividad son los diminutivos, tema del que nos ocuparemos en referencia al español ya que este idioma se caracteriza por el frecuente uso de estos sufijos apreciativos (Lázaro Mora, 1992: 4647). El presente artículo se centrará en las funciones de los diminutivos en la variedad mexicana. En su uso común dichos elementos están muy presentes y en ocasiones se llega incluso al abuso. Nuestro objetivo consiste en plantear un esquema propio de la clasificación semántico-pragmática del empleo de los diminutivos, apoyado en ejemplos extraídos de un corpus lingüístico de la Ciudad de México.

\section{Consideraciones teóricas}

Basándonos en las informaciones del Diccionario de la Lengua Española de 2014 (DLE) y del Diccionario de términos filológicos (Lázaro Carreter, 1990: 145), proponemos la siguiente definición de diminutivo: producto de la fusión de un lexema con uno o más sufijos diminutivos (p. ej.,-ito, -ico, -illo), el cual se caracteriza por una dualidad semántica: puede, por un lado, expresar una valoración afectiva, la cual, a su vez, puede referirse al tamaño - más concretamente a su disminución - , en cuyo caso es bastante objetiva, o, asimismo, transmitir una opinión subjetiva, que incluye una carga emocional y muestra cortesía, familiaridad, atenuación, ironía o menosprecio; puede, por otro lado, el diminutivo intensificar el mensaje denotado por el vocablo al que se agrega el sufijo diminutivo. 
Merece la pena aclarar que no en todas las áreas hispanohablantes la sufijación diminutiva se realiza de igual manera. Aparecen pues, en esta esfera, diferencias considerables entre la península ibérica e Hispanoamérica, resultantes de la elección de sufijos concretos, la frecuencia de uso y las clases de palabras a las que se agrega.

\subsection{El 'abuso' de los diminutivos en el español de México y posi- bles explicaciones de este fenómeno}

Como ya se ha mencionado, el abuso de los diminutivos es un rasgo particular de los mexicanos. Así pues, merece la pena reflexionar acerca de cuál es el motivo de este fenómeno. Después de analizar numerosas fuentes, se puede constatar que en el estilo mexicano de comunicación verbal han influido factores históricos, sociales y culturales. Dado que México fue una colonia española, el idioma español fue impuesto allí por los conquistadores (Lipski, 2009: 296-297): aunque la población indígena era mayoritaria, debido a su estatus más bajo en la jerarquía social, empezó a hablar la lengua de los colonizadores bajo su presión. Tanto en el pasado como hoy en día, en la sociedad mexicana existen notables diferencias económicas y sociales que provocan que los interlocutores mexicanos contemporáneos sigan prestando mucha atención al estatus social en los contactos personales (Curcó, 1998: 129-171; Walczak, 2007: 329-330), y por eso, con el fin de mostrar cortesía, utilizan múltiples diminutivos.

Según Dávila Garibi (1959: 91-94), la proyección de la multiplicidad de formas diminutivas usadas - e incluso abusadas - actualmente en México en la conversación familiar proviene de manera considerable de un impacto doble: el idioma de los conquistadores y la lengua de población indígena, en particular del náhuatl. El español mexicano adoptó la costumbre azteca de emplear con mucha frecuencia los diminutivos, cuya variedad era mucho más abundante que en el idioma de los invasores. Los síntomas del abuso de la diminutivización son, p. ej., la disminución, la cual se refleja en los adverbios, que normalmente son invariables (p. ej., adiosito, apenitas, por favorcito); en la palabra Diosito o Papá Diosito, derivada de Dios; o en extranjerismos 
que no se han adaptado al sistema fonológico español (p. ej., jolecito —de hall-o swettercito — de sweter-) (ibidem: 92).

\subsection{Funciones de los diminutivos en la lengua española}

Los análisis semántico-pragmáticos concernientes a los sufijos diminutivos en el español son objeto de numerosas polémicas entre los investigadores, que ponen de manifiesto distintos pareceres y proponen diversas y, a veces, similares interpretaciones de los valores en cuestión. Ya en la primera gramática del español, titulada Gramática castellana y publicada en 1492, Antonio de Nebrija, autor de la obra, afirma que los diminutivos constituyen una herramienta para expresar la idea de pequeñez (Nebrija, en: Náñez Fernández, 2006: 39). Por su parte, Andrés Bello y Rufino Cuervo (1954: 75-77) indican que la idea predominante expresada por los diminutivos es, al igual que lo que se sostiene en el trabajo de Nebrija, la de pequeñez, aunque añaden también otras ideas tales como cariño, compasión, desprecio y burla. Un punto de vista innovador, diferente de las opiniones de sus antecesores, lo presenta Amado Alonso (1974) al constatar que los diminutivos, desde el principio, han tenido un significado afectivo y no empequeñecedor. No renuncia por completo a la idea relativa a la dimensión, pero considera que la función disminuidora no aparece en el habla con una frecuencia más alta que en el lenguaje escrito como para ocupar el primer puesto.

\subsection{Objetivo del trabajo, descripción del corpus y método aplicado} Una vez planteado el marco teórico de la diminutivización en el español, podemos proceder a la presentación del objetivo principal de nuestro estudio: determinar con qué fin emplean los diminutivos los usuarios del español en la Ciudad de México.

El análisis lo hemos realizado basándonos en los ejemplos concretos extraídos del Corpus Sociolingüistico de la Ciudad de México (CSCM), accesible en línea ${ }^{1}$. En el análisis, además de los lingüísticos,

${ }^{1}$ Véase: www.lef.colmex.mx/index.php/investigaciones/corpussociolinguistico-de-la-ciudad-de-Mexico-cscm 
hemos prestado atención a factores sociolingüísticos como el sexo y el nivel de instrucción (superior, medio y bajo), los cuales pueden influir en el uso de diminutivos. A pesar de la gran cantidad de información incluida en el corpus sometido al análisis, debido a la extensión limitada del presente artículo, nos vemos obligados a aplicar ciertas delimitaciones. Hemos decidido analizar solamente los datos concernientes a un grupo de edad concreto: el de las personas jóvenes (36 personas) entre 20 y 34 años, ya que tienden a emplear con mayor frecuencia el lenguaje coloquial, que abunda más en formas diminutivas que el registro culto.

La fuente de inspiración para crear el esquema de clasificación del uso de los diminutivos a través del que hemos llevado a cabo el análisis del material investigado son los modelos propuestos por Gaarder (1966: 585-586) y Reynoso Noverón (2005: 80-84). Los factores que han influido en la elección de los esquemas planteados son los siguientes: su claridad y estructura lógica y el hecho de haber sido elaborados a partir de datos provenientes del español de México. Aparte de ello, estos esquemas constituyen una compilación de los valores diminutivos, que también asume nuestra definición del diminutivo.

Hemos distinguido cuatro categorías principales en la valoración de diminutivos: a las tres primeras, que coinciden con las que propone Reynoso Noverón (2005: 80-84) (las valoraciones cuantificadora, cualificadora y relacional) hemos añadido una cuarta categoría concerniente a los diminutivos multifuncionales. Como, tras el estudio preliminar del corpus, hemos concluido que las subcategorías que expresan afecto en el modelo de Reynoso Noverón son demasiado superficiales con referencia a la realidad lingüística, las hemos complementado con algunas funciones seleccionadas que destaca Gaarder (1966: 585-586). En la siguiente tabla presentamos el modelo según el cual hemos llevado a cabo nuestro análisis: 
1. VALORACIÓN CUANTIFICADORA (se refiere a la dimensión del objeto sometido a la diminutivización):

a) cuantificadora (objetiva; informa sobre la dimensión);

b) descentralizadora (subjetiva; reduce dimensión, extensión, cantidad, intensidad o duración de la palabra base y subraya los rasgos peyorativos; amortigua el significado de la palabra base que constituye el peor elemento en un grupo semántico);

c) centralizadora (subjetiva; incrementa dimensión, extensión, cantidad, intensidad o duración de la palabra base y realza los rasgos positivos; intensifica el significado del vocablo que se localiza en el centro de su grupo y es su mejor representante).

2. VALORACIÓN CUALIFICADORA (subjetiva; se refiere a cualidades que para el hablante constituyen el objeto sometido a la diminutivización y las emociones que produce en él):

a) negativa (menosprecio y desprecio, resultantes de odio, resentimiento, asco, etc.);

b) positiva (consideración, aprecio, afición, cariño).

3. VALORACIÓN RELACIONAL (muy subjetiva; se centra en las relaciones entre el hablante y el interlocutor y el objeto sometido a la diminutivización, la cercanía entre ellos y la afectividad; permite influir sobre el interlocutor con el fin de conseguir su reacción positiva o negativa):

a) irónica (el diminutivo es un recurso para lograr un efecto de burla, satírico);

b) amortiguadora (eufemística, cortés; el hablante intenta atenuar la información desagradable que recibe el interlocutor);

c) respetuosa (mediante la cortesía o humildad pretende lograr por parte del hablante la simpatía del interlocutor);

d) de familiaridad (incluyendo familiaridad impertinente; indica la cercanía y se caracteriza por la libertad, teniendo índole amistosa);

e) de apocamiento estratégico (engaño, falsa modestia, etc., a fin de conseguir un objetivo determinado);

f) lenguaje infantil y estudiantil (sirve para sustituir palabras inconvenientes; dirigido a los niños y utilizado por ellos, pero también, a veces, el lenguaje de los adultos se estiliza con este tipo de lengua).

4. DIMINUTIVOS MULTIFUNCIONALES (a la vez encadenan en sí diversos valores de las categorías susodichas). 


\section{Análisis del material}

La metodología de investigación se basa en un análisis cualitativo-cuantitativo desde un enfoque sincrónico. La selección de ejemplos ha sido subjetiva y la hemos realizado de tal manera que ponga de manifiesto la diversidad de funciones que cumplen los diminutivos. Debido a la limitada extensión del presente artículo, no comentaremos todos los ejemplos, sino únicamente los que parecen, a nuestro juicio, más interesantes para el propósito de este trabajo.

I: Él tenía como unos veintiséis años y no había hecho nada de su vida, era un relajo, no tenía trabajo, o sea, bueno, tenía trabajos eventuales, pero serios no.

E: Era un desmadre.

I: Tenía nada así como definido de "qué quiero hacer", ¿no?

E: Bueno, yo creo que es la inseguridad y otra cosa es en el hecho de que lo que tú hagas defina lo que tú eres como ser humano, ¿no?

I: Aparte era inseguro, era como un chavito (...) en muchos sentidos.

(CSCM, nivel superior, M, entrevista 7, ME-107-31M-00, p. 182)

Valoración cualificadora con función negativa: el diminutivo chavito se forma a partir del vocablo base chavo por la adición del sufijo -ito al sustantivo. La semántica del lexema chavo y del diminutivo chavito generalmente posee connotaciones neutrales o positivas; en cambio, en el ejemplo comentado el contexto situacional indica que es al revés: la persona de la que se habla infunde en el emisor emociones negativas. Le irrita la inmadurez del objeto disminuido, su inseguridad (que se manifiesta en la falta de ideas para la vida) y, en general, su comportamiento, más propio de un niño que de un adulto. El diminutivo usado en este contexto transmite menosprecio, desprecio, carencia de valor de la persona a la cual se refiere, por lo que, en este caso, observamos una valoración cualitativa cargada negativamente.

I: Pero todavía está chiquita, ay sí.

E: ¿Cuántos años tiene?

I: Catorce. 
E: Pues es una niña, está súper chiquita.

(CSCM, nivel superior, M, entrevista 7, ME-107-31M-00, p. 176)

Valoración relacional con función irónica: la informante define a su hermana adolescente con la palabra chiquita, con la que pretende referirse a su edad. Cuando se revela que es una persona de catorce años, el entrevistador sólo aparentemente da la razón a la informante y a su enunciado e incluye una burla hacia la expresión inadecuada de la edad de la persona aludida. El matiz irónico lo intensifica todavía más el vocablo acompañante: el adjetivo coloquial súper. El diminutivo se forma por la agregación del sufijo -ito al adjetivo chica; no obstante, solo el sufijo no es suficiente para descubrir las intenciones del emisor. En el marco de la valoración relacional cumple una función irónica con un matiz humorístico, lo cual ni siquiera se manifiesta claramente en el contexto de toda la situación comunicativa.

I: No me identifico con el "con tu granito de arena puedes cambiar el mundo", al menos ahorita no, a lo mejor en un futuro sí.

(CSCM, nivel superior, H, entrevista 5, ME-190-31H-05, p. 135)

Valoración relacional con función amortiguadora: el adverbio ahora y el sufijo -ita han servido para formar el diminutivo ahorita. Para comprender la función de este diminutivo es necesario atender a todo el contexto situacional. El informante abiertamente reconoce que no está de acuerdo con el dicho: "con tu granito de arena puedes cambiar el mundo"; no obstante, muestra que desconoce la opinión de su interlocutor acerca de esta cuestión y no desea causarle disgusto si este tuviera otro parecer, atenúa la impresión eventualmente negativa que puede provocar con su respuesta y, para suavizarla, aprovecha el diminutivo ahorita, que indica que algún día es posible que cambie de opinión respecto al tema sobre que discuten. El hablante trata de salvaguardar su imagen positiva con el fin de ser percibido positivamente, aunque, por otro lado, intenta no amenazar la imagen negativa del interlocutor y no imponerle su opinión.

I: Después se separaron, cuando falleció mi hermanito a los tres años. (CSCM, nivel bajo, M, entrevista 82, ME-302-11M-07, p. 327) 
Diminutivos multifuncionales con función cuantificadora y positiva: el derivado hermanito se ha creado mediante la adición del -ito al sustantivo hermano. Las funciones que cumple el diminutivo se deducen del contexto de enunciado; el informante habla de la muerte de su hermano de tres años; el diminutivo desempeña dos papeles: cuantificador (se refiere a la edad del niño) y positivo (muestra que el hermano fallecido ha tenido mucha importancia para el hablante y despierta en él sentimientos positivos).

I: Imagínate un danés, pues lo hablan en su paisito (...) pero salen y ya no se pueden comunicar.

(CSCM, nivel superior, $\mathrm{H}$, entrevista 4, ME-155-31H-01, p. 112)

Diminutivos multifuncionales con función negativa e irónica: al sustantivo país se ha agregado el sufijo -ito creando un diminutivo. La adición misma de -ito podría implicar la pequeña dimensión del país sobre el que trata el hablante, aunque en este caso no es así. Del contexto resulta que la forma diminutivizada posee dos matices: por un lado, cumple una función negativa (el hablante menosprecia el valor de un país con cuyo idioma sus hablantes solo pueden comunicarse en su territorio); pero, por otro lado, también manifiesta una burla que surte un efecto irónico.

I: (...) Ese día de la boda, sí, fue muy chistoso. A ver mis tres abuelas ahí juntas, porque realmente a las tres les digo abuelita (...).

(CSCM, nivel superior, H, entrevista 6, ME-197-31H-01, p. 143)

Diminutivos multifuncionales con función positiva y con función de familiaridad: el sustantivo abuela en unión con el sufijo -ita ha formado el diminutivo abuelita. Posee una función positiva e incluye en sí mismo una carga emocional que expresa cariño. Al igual que en muchos de los ejemplos anteriores, el contexto del enunciado llama la atención sobre otro papel del diminutivo, en este caso el de familiaridad, que puede deducirse del uso por el hablante de la denominación abuelita para referirse a tres personas cuando, normalmente, se tienen solo dos abuelas. Se implica de ese modo que hay una persona más con la que se mantienen relaciones cercanas y amistosas. 
I: ¿A qué hora te paraste?

E: ¿El domingo?

I: ¡El lunes, burrito!

E: Ah, ¿el lunes?, a las siete ya estaba parado, sí, sí, sí, tenía que llegar temprano.

(CSCM, nivel superior, H, entrevista 6, ME-197-31H-01, p. 163)

Diminutivos multifuncionales con función irónica y amortiguadora: el lexema burro tras la agregación del sufijo -ito se ha convertido en el diminutivo burrito. El significado real de este derivado podemos deducirlo solo a través del contexto situacional: el hablante se dirige a su interlocutor con la palabra burrito ya que este no ha entendido parcialmente una pregunta, lo cual resulta, probablemente, de la propia interacción comunicativa entre los participantes. Este diminutivo desempeña un papel irónico, dado que el hablante se burla, aunque de manera chistosa, del interlocutor, y, al mismo tiempo, mediante el empleo de dicho diminutivo, amortigua su enunciado con el fin de no ofender al interlocutor y no amenazar su imagen positiva; además, pretende proteger su propia faz y evitar que su interlocutor lo considere una persona descortés.

\section{Observaciones y conclusiones}

Como ya se ha mencionado, el análisis se ha realizado a base de las entrevistas llevadas a cabo con 36 personas de ambos sexos (18 mujeres y 18 hombres); en los enunciados de los informantes hemos encontrado un total de 1065 diminutivos. La interpretación de los valores de los diminutivos es un asunto muy complejo por su enorme subjetividad, por lo que nuestro estudio también se caracteriza por una subjetividad significativa, aunque hemos intentado abordar el tema que nos ocupa de la manera más objetiva posible. En relación a lo señalado, hemos de hacer hincapié en que a lo largo del proceso de evaluación de las funciones de los diminutivos no podemos guiarnos solamente por el sufijo agregado, sino que hay que tener en cuenta también la semántica del vocablo base, las palabras acompañantes y el contexto, todo lo 
cual es primordial para la interpretación correcta de la intención del hablante dado que un mismo vocablo, en situaciones diferentes, puede poseer distintos matices semánticos.

Uno de los objetivos del análisis ha sido verificar si nuestro esquema que ilustra la clasificación semántico-pragmática del uso de diminutivos es adecuado. Las investigaciones demuestran que es correcto ya que todos los diminutivos hallados en el corpus estudiado se pueden clasificar en las respectivas categorías funcionales: cuantificadora, cualificadora, relacional, o en varias a la vez, es decir, en la categoría multifuncional.

En otro orden de cosas, hemos observado que los diminutivos expresan principalmente afectividad (hemos censado 935 casos, lo cual constituye el $87,79 \%$ de todos los diminutivos que aparecen en el material de estudio). Por su parte, el diminutivo referido al tamaño del objeto aparece solo en 130 casos (el 12,21\% del número total de diminutivos). Una comparación más detallada, que tiene en cuenta todas las funciones de los diminutivos, se halla en la tabla que se ofrece a continuación:

\begin{tabular}{|l|c|c|c|c|c|}
\hline \multirow{4}{*}{ FUNCIÓN } & \multicolumn{5}{|c|}{$\begin{array}{c}\text { NÚMERO DE DIMINUTIVOS EMPLEADOS } \\
\text { SEGÚN EL NIVEL DE INSTRUCCIÓN }\end{array}$} \\
\cline { 2 - 6 } & $\begin{array}{c}\text { SUPE- } \\
\text { RIOR }\end{array}$ & MEDIO & BAJO & \multicolumn{2}{|c|}{ TOTAL } \\
\cline { 2 - 6 } & Cantidad & Cantidad & Cantidad & Cantidad & $\%$ \\
\hline CUANTIFICADORA & 45 & 39 & 46 & 130 & 12,21 \\
\hline $\begin{array}{l}\text { DESCENTRALIZA- } \\
\text { DORA }\end{array}$ & 24 & 17 & 71 & 112 & 10,52 \\
\hline CENTRALIZADORA & 131 & 156 & 202 & 489 & 45,92 \\
\hline NEGATIVA & 11 & 2 & 0 & 13 & 1,22 \\
\hline POSITIVA & 13 & 47 & 6 & 66 & 6,20 \\
\hline IRÓNICA & 9 & 3 & 3 & 15 & 1,41 \\
\hline AMORTIGUADORA & 46 & 16 & 22 & 84 & 7,89 \\
\hline RESPETUOSA & 5 & 1 & 2 & 8 & 0,75 \\
\hline FAMILIARIDAD & 12 & 0 & 2 & 14 & 1,31 \\
\hline
\end{tabular}




\begin{tabular}{|l|c|c|c|c|c|}
\hline $\begin{array}{l}\text { APOCAMIENTO } \\
\text { ESTRATÉGICO }\end{array}$ & 0 & 0 & 0 & 0 & 0 \\
\hline $\begin{array}{l}\text { LENGUAJE INFAN- } \\
\text { TIL Y ESTUDIANTIL }\end{array}$ & 7 & 3 & 2 & 12 & 1,13 \\
\hline $\begin{array}{l}\text { MULTIFUNCIONA- } \\
\text { LES: }\end{array}$ & 23 & 48 & 50 & 122 & 11,44 \\
\hline cuantificadora + positiva & 8 & 3 & 8 & 19 & 1,78 \\
\hline $\begin{array}{l}\text { descentralizadora + } \\
\text { negativa }\end{array}$ & 0 & 2 & 0 & 2 & 0,19 \\
\hline centralizadora + positiva & 2 & 26 & 12 & 40 & 3,76 \\
\hline centralizadora + irónica & 0 & 0 & 1 & 1 & 0,09 \\
\hline $\begin{array}{l}\text { centralizadora + amorti- } \\
\text { guadora }\end{array}$ & 1 & 0 & 0 & 1 & 0,09 \\
\hline $\begin{array}{l}\text { centralizadora + respe- } \\
\text { tuosa }\end{array}$ & 1 & 0 & 0 & 1 & 0,09 \\
\hline negativa + irónica & 1 & 0 & 0 & 1 & 0,09 \\
\hline $\begin{array}{l}\text { negativa + amortigua- } \\
\text { dora }\end{array}$ & 0 & 1 & 0 & 1 & 0,09 \\
\hline $\begin{array}{l}\text { positiva + amortigua- } \\
\text { dora }\end{array}$ & 2 & 0 & 0 & 2 & 0,19 \\
\hline positiva + respetuosa & 4 & 4 & 29 & 37 & 3,47 \\
\hline positiva + familiaridad & 1 & 6 & 0 & 7 & 0,66 \\
\hline $\begin{array}{l}\text { positiva + lenguaje } \\
\text { infantil }\end{array}$ & 0 & 4 & 0 & 4 & 0,38 \\
\hline \begin{tabular}{l} 
irónica + amortiguadora \\
\hline $\begin{array}{l}\text { amortiguadora + apoca- } \\
\text { miento estratégico }\end{array}$
\end{tabular} & 1 & 2 & 0 & 3 & 0,28 \\
\hline $\begin{array}{l}\text { familiaridad + lenguaje } \\
\text { infantil }\end{array}$ & 2 & 0 & 0 & 2 & 0,19 \\
\hline SUMA: & 326 & 333 & 406 & 1065 & 100 \\
\hline
\end{tabular}

Tabla 1. Número de diminutivos según el nivel de instrucción ${ }^{2}$

Las estadísticas ponen de manifiesto que en enunciados de representantes de ambos sexos y de todos los niveles de instrucción predomina la función centralizadora de los diminutivos (encontramos en

\footnotetext{
${ }^{2}$ Tabla de elaboración propia.
} 
mujeres 246 casos —el 43,08\% de los diminutivos usados por ellas-, mientras que en hombres se dan 242 casos-el 46,38\% de los derivados utilizados por ellos-). La única función que no ha aparecido en nuestro material es la de apocamiento estratégico. Sin embargo, cabe precisar que está presente como componente de los diminutivos multifuncionales. Estos últimos son utilizados con bastante frecuencia por mujeres ( 83 veces $-14,50 \%$ de todos los diminutivos femeninos-). Además, en nuestro análisis hemos observado 15 pares funcionales de diminutivos.

La función cuantificadora aparece a través de la unión con la positiva, mientras que la descentralizadora concurre con la negativa. Por su parte, la centralizadora se une con cuatro funciones diferentes, a saber: la positiva, la irónica, la amortiguadora y la respetuosa. Además, la función negativa se une indistintamente a la irónica y a la amortiguadora. Por otro lado, la positiva aparece junto con las funciones amortiguadora, respetuosa, la de familiaridad y la de lenguaje infantil. Asimismo, la función irónica coexiste con la amortiguadora y esta última se une con el apocamiento estratégico. Otra combinación que hemos encontrado es la de familiaridad con la de lenguaje infantil.

Las frecuencias más altas de uniones entre funciones son las siguientes: función centralizadora con positiva, 40 casos —el 3,76\%-; positiva con respetuosa, 37 -el 3,47\%-; y cuantificadora con positiva, 19 - el 1,78\% - Un hecho digno de mención a este respecto es que las fronteras, en teoría bien visibles, entre ciertas funciones en la práctica a veces se difuminan, lo que crea dificultades para determinar un valor real. Tal casuística viene provocada principalmente por las funciones descentralizadora y negativa junto con la centralizadora y la positiva.

De entre los nueve sufijos diminutivos de la lengua española definidos por la Real Academia Española (RAE 2010: 627), en la parte del corpus que hemos analizado se han dado solamente cuatro para formar los diminutivos: -ito $(1024-96,15 \%$ - ) ; -illo $(37-3,47 \%$ - ) - -ete $(3-0,28 \%-)$ e -ín $(1-0,10 \%-)$. Todos los sufijos enumerados han aparecido en enunciados de personas de nivel de instrucción superior. 
Los informantes que poseen una formación media o baja han usado los diminutivos creados mediante sólo dos sufijos: -ito e -illo.

\section{FRECUENCIA DE USO DE LOS SUFIJOS DIMINUTIVOS}

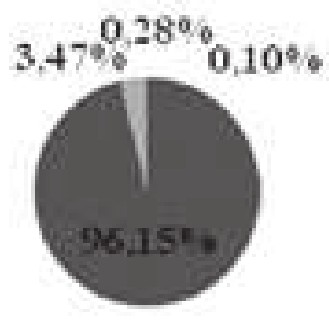

Gráfico 1. Frecuencia de uso de los sufijos diminutivos (todos los niveles de instrucción $)^{3}$

El análisis pone de manifiesto que, entre las clases de palabras compatibles con los sufijos diminutivos, la mayor presencia la tienen los adverbios (558 veces - 52,39\%-), a continuación, los sustantivos (361 casos - 33,90\%-) y, finalmente, los adjetivos (146 ocurrencias $-13,71 \%-)$.

\section{COMPATIBILIDAD DE LAS PARTES DE LA ORACIÓN CON SUFIJOS DIMINUTIVOS}

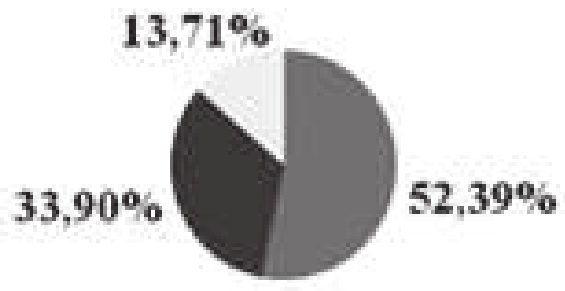

EADVERBIOS

- SUSTANTIVOS

ADJETIVOS

Gráfico 2. Compatibilidad de las partes de la oración con sufijos diminutivos (todos los niveles de instrucción) ${ }^{4}$

${ }^{3}$ Gráfico de elaboración propia.

${ }^{4}$ Gráfico de elaboración propia. 
En referencia a los adverbios, un ejemplo muy interesante lo constituye el diminutivo ahorita, que es el que presenta mayor frecuencia de entre todos los adverbios. El análisis muestra que este vocablo puede poseer tres valores: centralizador, amortiguador y descentralizador.También puede tener carácter polisémico al unir el valor amortiguador con el centralizador o el amortiguador con el descentralizador. La palabra ahorita se encuentra en el Diccionario del español de México, según el cual es un adverbio coloquial que significa 'ahora, en este momento, dentro de poco tiempo'. Esto indicaría que es una palabra lexicalizada. No obstante, nuestras investigaciones revelan que ahorita cuenta con ciertos valores típicos de los diminutivos. Por ello, no estamos en situación de constatar inequívocamente si se trata de un vocablo completamente lexicalizado o de un diminutivo. Quizá se halle en la fase final del proceso de lexicalización y en poco tiempo se convierta en el equivalente de la palabra ahora o, incluso, la sustituya en el español de México. Sin embargo, también es posible que la definición del diccionario, en cierto grado, sea inadecuada al señalar 'ahora' como una de las acepciones de ahorita, de lo cual podemos inferir que la lexicografía a veces no puede seguir el paso de las realidades lingüísticas contemporáneas.

Acerca de los factores sociolingüísticos, podemos afirmar que tanto el sexo como el nivel de instrucción de los hablantes desempeñan un papel relevante en el campo de estudio que estamos abordando. Como pone de manifiesto el análisis realizado, las mujeres utilizan los diminutivos más frecuentemente que los hombres; en sus enunciados hemos encontrado 571 diminutivos $(53,62 \%)$, mientras que en los enunciados masculinos aparecen 494 diminutivos (46,38\%). Las diferencias parecen no ser demasiado significativas, aunque a la hora de explicarlas podemos atribuir tal situación al hecho de que las mujeres son más expresivas, por lo que su lenguaje es más rico en expresiones afectivas. La mayoría de los diminutivos con connotaciones positivas son utilizados por mujeres, mientras que los derivados que se asocian con connotaciones negativas se dan en cantidades parecidas en los enunciados de ambos sexos. 


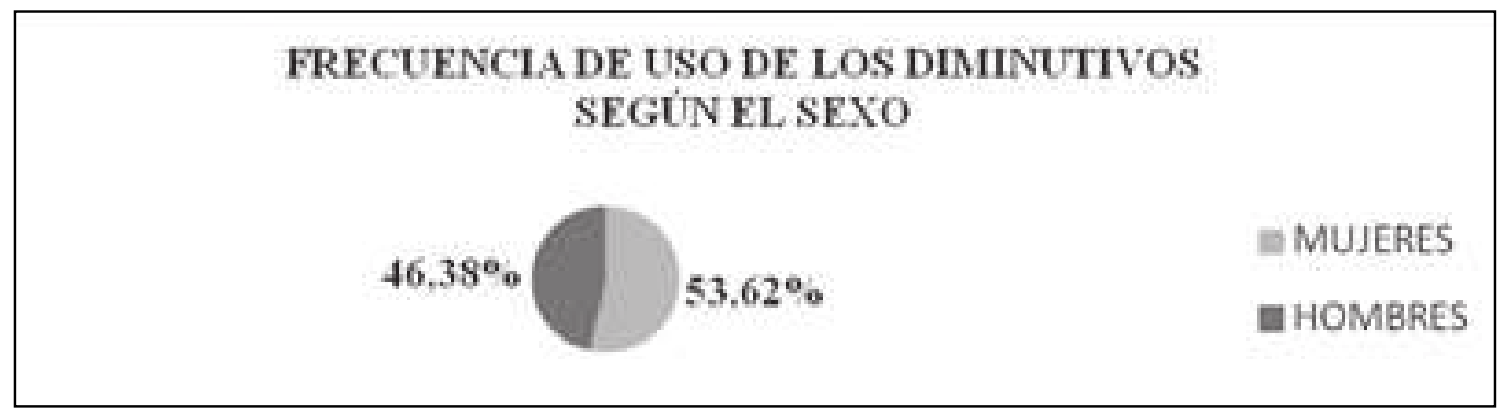

Gráfico 3. Frecuencia de uso de los diminutivos según el sexo (todos los niveles de instrucción $)^{5}$

Las personas con un nivel de instrucción bajo usan con mayor asiduidad los diminutivos porque suelen emplear el lenguaje coloquial. Nuestras estadísticas señalan que los representantes de este nivel de formación han empleado los diminutivos 406 veces $(38,12 \%)$; en cambio, las personas con instrucción media los usan 333 veces $(31,28 \%)$ y las que cuentan con instrucción superior 326 veces $(0,60 \%)$. Un hecho colegible de estos datos es que las personas con instrucción superior dan mayor importancia al empleo del lenguaje estándar neutro.

Se puede deducir que cuanto más alto es el nivel de formación, más alto es el registro lingüístico, lo cual se refleja en la conciencia de la cortesía verbal (el número de los diminutivos amortiguadores es mucho mayor entre las personas que han recibido este tipo de educación [46] que en los enunciados producidos por las personas de instrucción baja [22] y media [16]).

${ }^{5}$ Gráfico de elaboración propia. 


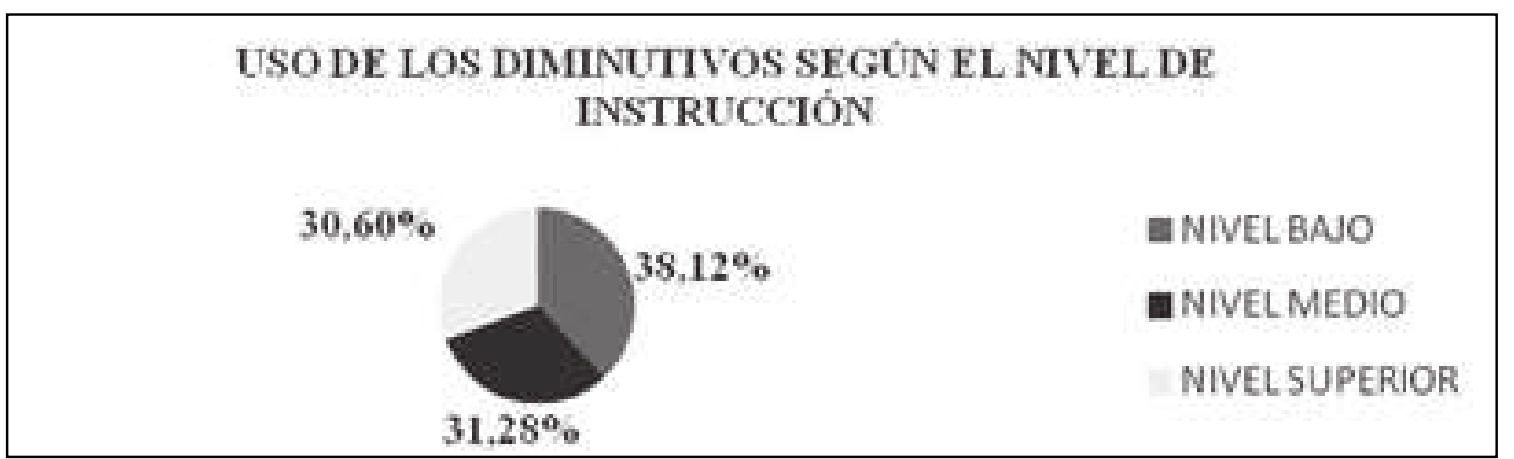

Gráfico 4. Frecuencia de uso de los diminutivos según el nivel de instrucción (todos los niveles de instrucción) ${ }^{6}$

Así pues, nuestro trabajo viene a confirmar la hipótesis de que los mexicanos tienden a abusar de los diminutivos. Sin embargo, se puede ver este fenómeno desde otra perspectiva y suponer que no se trata de un abuso lingüístico, sino que estamos ante cierto rasgo específico de la variante mexicana del idioma español. En este caso, el vocablo abuso no es ni negativo ni positivo en sí, dado que pierde su valor axiológico y se convierte en una palabra de carácter neutro.

\section{Bibliografía}

ALONSO, A. (1974), Estudios lingüisticos. Temas españoles, Gredos, Madrid.

BELLO, A., CUERVO, R. J. (1978), Gramática de la lengua castellana, Editorial de Ciencias Sociales, La Habana.

BROWN, P., LEVINSON, S. (1987), Politeness. Some Universals in Language Usage, Cambridge University Press, Cambridge.

CURCÓ, C. (1998), “'No me harías un favorcito?: reflexiones en torno a la expresión de la cortesía verbal en el español de México y el español peninsular", en: Haverkate, H., Mulder, G., Fraile Maldonado, C. (eds.), La pragmática lingüistica del español. Recientes desarrollos, Brill, Amsterdam-Atlanta, GA, pp. 129-171.

\footnotetext{
${ }^{6}$ Gráfico de elaboración propia.
} 
DÁVILA GARIBI, J. I. (1959), "Posible influencia del náhuatl en el uso y abuso del diminutivo en el español de México", Estudios de cultura náhuatl, 1, pp. 91-94 [on-line] http://www.historicas.unam.mx/publicaciones/revistas/nahuatl/pdf/ecn01/007.pdf, 03.2015.

GAARDER, B. A. (1966), "Los llamados diminutivos y aumentativos en el español de México", Publications of the Modern Language Association of America, 81 (7), pp. 585-595, [on-line] http://doi.org/10.2307/461214. LÁZARO MORA, F. A. (1992), "La derivación apreciativa", en: Bosque, I., Demonte, V. (dirs.), Gramática descriptiva de la lengua española, t. 3, Espasa-Calpe, Madrid, pp. 4645-4682.

LIPSKI, J. M. (2009), El español de América, trad. S. Iglesias Recuero, Cátedra, Madrid.

NÁÑEZ FERNÁNDEZ, E. (2006), El diminutivo. Historia y funciones en el español clásico y moderno, Ediciones de la Universidad Autónoma de Madrid, Madrid.

Real Academia Española, Asociación de Academias de la Lengua Española (2010), Nueva gramática de la lengua española. Morfología, Sintaxis I, Espasa Libros, Madrid.

REYNOSO NOVERÓN, J. (2005), "Procesos de gramaticalización por subjetivización: el uso del diminutivo en español”, en: Eddington, D. (ed.), Selected Proceedings of the 7th Hispanic Linguistics Symposium, Cascadilla Proceedings Project, Somerville, MA, pp. 76-89, [on-line] www. lingref.com/cpp/hls/7/paper1088.pdf, 04.2015.

WALCZAK, G. (2007), “Grzeczność meksykańska, Zachować twarz rozmówcy”, en: Marcjanik M. (red.), Grzeczność na krańcach świata, Wydawnictwa Akademickie i Profesjonalne, Warszawa, pp. 329-341.

\section{Diccionarios}

DEM = Diccionario del español de México, [on-line] http://www.dem.colmex.mx (2016).

DLE = Diccionario de la Lengua Española, ed. 23a, [on-line] http://www. dle.rae.es, (2014-2016)

LÁZARO CARRETER, F. (1990), Diccionario de términos filológicos, Gredos, Madrid. 


\section{Corpus analizados:}

CSCM = Corpus Sociolingüístico de la Ciudad de México [on-line] www.lef.colmex.mx/index.php/investigaciones/corpus-socio linguistico-de-la-ciudad-de-Mexico-cscm

BUTRAGUEÑO, P. M., RASTRA, Y. (coords.), Materiales de PreseeaMéxico. Introducción a los materiales de la Ciudad de México. Nivel superior, vol. 1: Hablantes de instrucción superior, El Colegio de México, [on-line] www.lef.colmex.mx/sociolinguistica/CSCM/Introducciona los materiales de la Ciudad de Mexico nivel superior.pdf

BUTRAGUEÑO, P. M., RASTRA, Y. (coords.), Materiales de Preseea-México. Introducción a los materiales de la ciudad de México nivel medio, vol. 2: Hablantes de instrucción media, El Colegio de México, [on-line] www.lef.colmex.mx/sociolinguistica/CSCM/Introducciona los materiales de la Ciudad de Mexico nivel medio.pdf 